\title{
Neutrophil-to-lymphocyte ratio and chemotherapy response score as prognostic markers in ovarian cancer patients treated with neoadjuvant chemotherapy
}

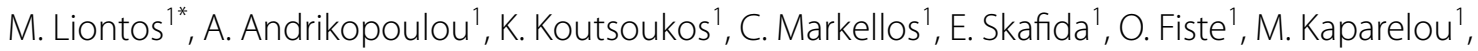 \\ N. Thomakos' ${ }^{2}$ D. Haidopoulos ${ }^{2}$, A. Rodolakis ${ }^{2}$, M. A. Dimopoulos ${ }^{1}$ and F. Zagouri ${ }^{1}$
}

\begin{abstract}
Background: Neoadjuvant chemotherapy (NACT) followed by interval debulking surgery (IDS) is the recommended approach in patients with advanced epithelial ovarian cancer (EOC). However, most patients eventually relapse despite the initial high response rate to chemotherapy. Neutrophil-to-lymphocyte ratio is a well-known biomarker that reflects severe inflammation, critical illness, and mortality in various diseases. Chemotherapy response score (CRS) and neutrophil-to-lymphocyte ratio (NLR) have been identified as potential biomarkers of platinum resistance and disease prognosis. We retrospectively evaluated 132 patients with stage IIIc or IV ovarian/fallopian tube/primary peritoneal cancer who had received NACT followed by IDS from 01/01/2003 to 31/12/2018. CRS was assessed on omental specimens collected from IDS according to ICCR guidelines.
\end{abstract}

Results: Median age was 64.57 years (SD: 9.72; range 39.2-87.1). Most ovarian tumors were serous epithelial (90.9\%; 120/132). An elevated NLR (defined as > 3) was observed in 72\% (95/132) of patients in contrast with 28\% (37/132) of patients characterized by low NLR status. Median PFS (mPFS) and median overall survival (mOS) were 13.05 months (95\% Cl: 11.42-14.67)) and 34.69 months (95\% Cl: 23.26-46.12) respectively. In univariate analysis, CRS3 score was significantly associated with prolonged mPFS (CRS1/2: 12.79 months vs CRS3: 17.7 months; $P=0.008$ ). CRS score was not associated with mOS $(P=0.876)$. High NLR was not significantly associated with mPFS $(P=0.128)$, however it was significantly associated with poor mOS $(P=0.012)$. In multivariate analysis, only performance of surgery maintained its statistical significance with both PFS $(P=0.001)$ and OS $(P=0.008)$.

Conclusion: NLR could serve as a useful predictor of OS but not PFS in ovarian cancer patients receiving NACT. In accordance with our previous study, CRS score at omentum was found to be associated with PFS but not OS in ovarian cancer patients treated with NACT and IDS.

\footnotetext{
*Correspondence: mlionto@med.uoa.gr

${ }^{1}$ Department of Clinical Therapeutics, Alexandra General Hospital, School

of Medicine, National and Kapodistrian University of Athens, Athens,

Greece

Full list of author information is available at the end of the article
}

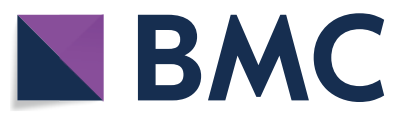

(c) The Author(s) 2021. Open Access This article is licensed under a Creative Commons Attribution 4.0 International License, which permits use, sharing, adaptation, distribution and reproduction in any medium or format, as long as you give appropriate credit to the original author(s) and the source, provide a link to the Creative Commons licence, and indicate if changes were made. The images or other third party material in this article are included in the article's Creative Commons licence, unless indicated otherwise in a credit line to the material. If material is not included in the article's Creative Commons licence and your intended use is not permitted by statutory regulation or exceeds the permitted use, you will need to obtain permission directly from the copyright holder. To view a copy of this licence, visit http://creativecommons.org/licenses/by/4.0/. The Creative Commons Public Domain Dedication waiver (http://creativeco mmons.org/publicdomain/zero/1.0/) applies to the data made available in this article, unless otherwise stated in a credit line to the data. 


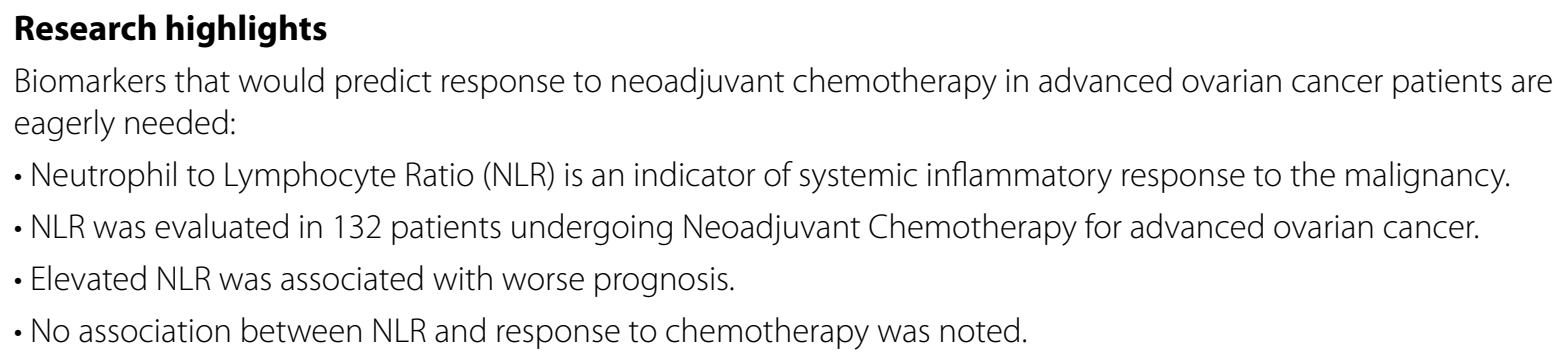

\section{Background}

Ovarian cancer is the eighth most common cause of cancer-related mortality in women globally and the leading cause of gynecological cancer-associated mortality in developed countries [1]. It is estimated that in 2020, about 308,069 new cases of ovarian cancer will be diagnosed and approximately 193,811 deaths will be reported worldwide [2]. Epithelial ovarian cancer (EOC) accounts for the $90 \%$ of ovarian cancers with high-grade serous ovarian carcinoma consisting the main histological subtype [3]. Despite the progress of cancer treatment during the last decade, over than two thirds of EOC cases are diagnosed at an advanced stage resulting in a 5-year survival rate of only $25 \%$ in stage III and IV disease [4]. The recommended approach for advanced-stage EOC is primary debulking surgery (PDS) followed by platinumbased chemotherapy. However, neoadjuvant chemotherapy (NACT) followed by interval debulking surgery (IDS) proved to be non-inferior to primary debulking surgery in stage III/IV patients unable to undergo optimal cytoreduction during PDS [5-9]. NACT approach consists of three or four cycles of upfront chemotherapy followed by IDS with the goal of complete resection. Despite the initial response to chemotherapy $(\sim 85 \%)$, most patients eventually relapse [10]. Therefore, there is an emerging need for markers and prognostic factors evaluating the response to treatment.

Chemotherapy response score (CRS) is a three-tier histopathological scoring system for validating response to NACT [11]. Patients that achieve CRS3 after NACT have improved progression free survival (PFS) and Overall Survival (OS) according to several retrospective studies and their metanalysis [12-23]. On that basis, the International Collaboration on Cancer Reporting (ICCR) incorporated the use of the CRS system in the current ovarian cancer histopathology reporting guide [13]. It would be of interest though to identify biomarkers that could predict which patients will or will not achieve CRS3 after NACT. That would allow patient selection through clinical trials for alternative therapeutic approaches aiming at improving dismal prognosis of these patients.
Recently, our group has shown that lymphocytic infiltration in pre-chemotherapy biopsies is associated with CRS3 after NACT. This indicates that response to chemotherapy could be associated with the immunological response and the inflammatory milieu of the tumor. Neutrophil-to-lymphocyte ratio (NLR) that serves as a measure of systemic inflammatory response is a prognostic biomarker that has been widely explored in oncology. NLR is easily measured through a complete blood count and could be determined under most circumstances. In ovarian cancer patients, there is evidence supporting preoperative NLR clinical utility not only in outcome prediction [24-32] but also in distinguishing benign from malignant ovarian tumors [33-35]. NLR has been associated with CD4+ helper T cells (Th17) count and IL-8 and IL-6 plasma concentration [36, 37], levels of stromal IL-2 [38] and serum fibrinogen [39]. Overall, higher NLR values positively correlated with platinum resistance and poor prognosis.

In this context, we evaluated the predictive and prognostic role of NLR in advanced ovarian cancer patients treated with NACT followed by IDS and examined its association with CRS.

\section{Methods}

We retrospectively identified patients with stage IIIc or IV ovarian/fallopian tube/primary peritoneal cancer who had received NACT followed by IDS during a 15-year period (January 2003-December 2018) in our institutional database. Patients with coexisting hematological malignancies, systemic administration of corticosteroids or any inflammatory conditions that could affect blood counts were excluded. Our institution has been certified by the European Society of Gyenocologic Oncology (ESGO) as a center of excellence for the treatment of ovarian cancer. The study has been performed in accordance with the 1964 Helsinki Declaration and has been approved by the Institutional Review Board of Alexandra University Hospital (Protocol Number: 513/1507-2020). Patients were selected for NACT and IDS if it was judged by the experienced Gynecologic Oncologists that they could not be debulked upfront to no residual 
tumor. Assessment involved imaging studies and/or laparoscopic evaluation. At least 3 cycles of NACT and adequate pathology specimens were required for inclusion to the analysis. IDS was performed at 4-6weeks from last dose of chemotherapy. Since 2013, that bevacizumab administration in the frontline setting became the standard of care in the country, all patients were planned to receive bevacizumab post IDS, unless contraindicated. All women had provided informed consent for their treatment as well as for their use of medical records for research purposes. Clinicopathological characteristics including age at diagnosis, stage and histology of the disease, CA125 at diagnosis, residual disease at IDS, BRCA mutation status, type of chemotherapy administered, neutrophil and lymphocyte count prior to neoadjuvant chemotherapy, progression of the disease and overall survival were collected from the medical records of the patients. Optimal debulking was defined as maximum residual tumor $<1 \mathrm{~cm}$ in diameter after IDS.

\section{Pathology review}

Tissue slides collected from IDS were thoroughly reviewed by an experienced gynecologic pathologist trained in assessment of Bohm's score (MS). CRS was assessed on omental specimens according to ICCR guidelines [13].

\section{Neutrophil to lymphocyte ratio evaluation}

Complete blood cell counts (CBC) were collected from all patients prior to commencing chemotherapy. NLR was defined as the absolute neutrophil count divided by the absolute lymphocyte count. An elevated NLR was defined as an NLR $>3$ based on previous studies [40-44].

\section{Statistical analysis}

Continuous variables were summarized with the use of descriptive statistical measures [median and percentiles (25th,75th)] and categorical variables were displayed as frequency tables (N, \%). The outcome of the debulking surgery was classified as complete (no residual disease) or optimal (residual disease below $1 \mathrm{~cm}$ ). Overall Survival (OS) was defined as the time between the start of chemotherapy and the date of death from any cause. Progression-free Survival (PFS) was defined as the time between the start of chemotherapy and the date of progression. Alive patients were censored at the date of last contact. Kaplan-Meier estimates were used to describe and visualize the effect of categorical variables on OS and PFS. Log-rank tests were used to explore the prognostic value of categorical variables on clinical outcomes. The association of these factors with OS was assessed through HRs and their 95\% confidence intervals estimated from univariate Cox proportional hazards models.
Interactions between covariates and time varying effects were studied. Multivariate Cox-regression model of CRS omentum adjusted for age, Stage, and Debulking surgery was presented. Proportional hazards $(\mathrm{PH})$ assumption was graphically assessed using plots of $-\ln \{-\ln ($ survival $)\}$ curves for each category of the covariates versus ln (analysis time). All statistical analyses were performed using the STATA/SE 16.0 software (Copyright 1985-2019 StataCorp LP).

\section{Results}

\section{Patient baseline characteristics}

Overall, 132 ovarian cancer patients treated with NACT were identified. Demographic, clinical and pathologic characteristics are presented in Table 1 . In brief, median age was 64.57 years (SD: 9.72; range 39.2-87.1). Most ovarian tumors were serous epithelial (90.9\%; 120/132), while there were isolated cases of endometrioid $(0.8 \%$; $1 / 132)$, poorly differentiated $(1.5 \% ; 2 / 132)$ tumors and nine cases $(6.8 \%)$ where histological subtype was not specified. Ninety patients $(68.2 \%)$ had stage IIIC disease and 41 patients $(31.1 \%)$ had stage IV disease.

A significant percentage of patients had performance status (PS) $\leq 1$ in our cohort (ECOG 0: 38.6\%; 51/132 and ECOG 1: $31.8 \%$; $42 / 132$ respectively). Overall, $78 \%$ $(103 / 132)$ of patients treated with three or four cycles of NACT eventually underwent IDS. The remaining 29 patients (22\%) never received a debulking cytoreductive surgery either due to rapid clinical deterioration and death prior to surgery, failure to achieve a disease regression to a surgically resectable extent or due to patients' preference. Debulking was optimal in 58.2\% (60/103) of the cases and suboptimal in $32 \%(33 / 103)$. Initial chemotherapy consisted of paclitaxel/carboplatin in the majority of cases $(85.6 \% ; 113 / 132)$ while the remaining received carboplatin monotherapy. Testing for BRCA1/2 mutations was conducted in $34.8 \%$ of cases (46/132). BRCA mutations were detected in 11 cases $(8.3 \%)$. Bevacizumab was administered in $31.8 \%(42 / 132)$ of patients either as first line maintenance treatment or as a subsequent line.

CRS score was available in 73 cases. 19 (26\%), $31(42.5 \%)$ and $23(31.5 \%)$ patients had ovarian CRS of 1 , 2 and 3 respectively. Patients were classified in two distinct groups: one group with CRS1/2 (68.5\%; 50/73) versus those with CRS3 (31.5\%; 23/73).

\section{NLR and its assocations with clinical characteristics in ovarian cancer}

Median value of neutrophil-to-lymphocyte ratio (NLR) was 4.18 (SD: 3.38; range 1.1-23.8). An elevated NLR (defined as $>3$ ) was observed in $72 \%(95 / 132)$ of patients in contrast with $28 \%(37 / 132)$ of patients characterized by low NLR status. Attempting to dissect the population 
Table 1 Baseline characteristics

\begin{tabular}{|c|c|}
\hline Characteristics & No. of patients (\%) \\
\hline \multicolumn{2}{|l|}{ Age (yr) } \\
\hline Median & 64.57 \\
\hline Range & $39.2-87.1$ \\
\hline \multicolumn{2}{|l|}{ Histological type } \\
\hline HGSC & 120 (90.9\%) \\
\hline Endometrioid & $1(0.8 \%)$ \\
\hline Undifferentiated & $2(1.5 \%)$ \\
\hline Not specified & $9(6.8 \%)$ \\
\hline \multicolumn{2}{|l|}{ FIGO stage } \\
\hline IIIC & $90(68.2 \%)$ \\
\hline IV & $41(31.1 \%)$ \\
\hline Not specified & $1(0.8 \%)$ \\
\hline \multicolumn{2}{|l|}{ Regimen of NAC } \\
\hline Taxane+carboplatin & $113(85.6 \%)$ \\
\hline Other & $16(12.1 \%)$ \\
\hline Not specified & $3(2.3 \%)$ \\
\hline \multicolumn{2}{|l|}{ Debulking surgery } \\
\hline Yes & $103(78 \%)$ \\
\hline No & $29(22 \%)$ \\
\hline \multicolumn{2}{|l|}{ ECOG score } \\
\hline $0-1$ & $93(70.4 \%)$ \\
\hline $2-3$ & $25(18.9 \%)$ \\
\hline 4 & $2(1.5 \%)$ \\
\hline Not specified & $12(9.1 \%)$ \\
\hline \multicolumn{2}{|l|}{ NLR } \\
\hline$\leq 3$ & $37(28 \%)$ \\
\hline$>3$ & $95(72 \%)$ \\
\hline \multicolumn{2}{|l|}{ CRS } \\
\hline CRS1/2 & $50(68.5 \%)$ \\
\hline CRS3 & $23(31.5 \%)$ \\
\hline \multicolumn{2}{|l|}{ Debulking } \\
\hline Optimal & $60(58.2 \%)$ \\
\hline Suboptimal & $33(32 \%)$ \\
\hline Not specified & $10(9.7 \%)$ \\
\hline \multicolumn{2}{|l|}{ BRCA testing } \\
\hline Yes & $46(34.8 \%)$ \\
\hline No & $85(64.4 \%)$ \\
\hline Not specified & $1(0.8 \%)$ \\
\hline \multicolumn{2}{|l|}{ BRCA mutation } \\
\hline Yes & $11(8.3 \%)$ \\
\hline No & $36(27.3 \%)$ \\
\hline Unknown & $85(64.4 \%)$ \\
\hline \multicolumn{2}{|l|}{ Bevacizumab treatment } \\
\hline Yes & $42(31.8 \%)$ \\
\hline No & $74(56.1 \%)$ \\
\hline Not specified & $16(12.1 \%)$ \\
\hline
\end{tabular}

of patients with high NLR, we evaluated the association of NLR with clinicopathological factors. High NLR was significantly associated with poor performance status (ECOG-PS $\geq 2)(P=0.001)$, but not stage $(P=1.000)$ or age $(P=0.441)$. In relation to the applied treatments, high NLR was not associated with monotherapy carboplatin as initial chemotherapy $(P=0.380)$ but was statistically significantly correlated with inability to perform debulking surgery $(P=0.019)$, and omittion of bevacizumab as a maintenance treatment $(P=0.004)$. For the 73 cases that CRS data were available, no statistical significant association with NLR was shown $(P=0.321)$.

\section{Survival analysis}

Overall, median PFS (mPFS) was 13.05 months (95\% CI: 11.42-14.67). In univariate analysis, NLR was not significantly associated with mPFS $(P=0.128)$ (Fig. 1$)$. mPFS was 14.56 months in patients with low NLR $(\mathrm{NLR}<3)$ (95\% CI: $11.44-17.67)$ in contrast to 12.39 months $(95 \%$ CI: 10.64-14.13) in the high NLR population. In contrast, CRS3 score was significantly associated with prolonged mPFS (CRS1/2: 12.79 months vs CRS3: 17.7 months; $P=0.008)$, along with other clinical parameters and treatment characteristics such as performance of cytoreductive surgery $(P<0.001)$, bevacizumab administration $(P=0.02)$, ECOG performance status $<2(P=0.061)$ and presence of BRCA $1 / 2$ mutations $(P=0.003)$ (Fig. 2$)$.

In the total population, median overall survival (mOS) was 34.69 months (95\% CI: 23.26-46.12). High NLR status was significantly associated with poor mOS $(P=0.012)$. Median OS was 47.18 months for patients with low NLR values before chemotherapy initiation (95\% CI: 32.77-61.59; SD: 7.35) versus 30.59 months in patients with high NLR (95\% CI: 23.36-37.82; SD: 3.68) (Fig. 3).

In univariate analysis, CRS score was not associated with mOS $(P=0.876)$ (Fig. 4). Performance status (ECOG $\leq 1)(P<0.001)$, bevacizumab administration $(P<0.001)$ and performance of cytoreductive surgery $(P<0.001)$ demonstrated a strong correlation with mOS. In contrast, age over $65(P=0.129)$ and quality of debulking $(P=0.568)$ was not significantly associated with mOS.

On multivariate analysis, the performance of surgery was the single factor statistically significantly associated with both PFS $(P=0.001)$ (Table 2$)$ and OS $(P=0.008)$ (Table 3). NLR failed to maintain its statistical significance on multivariate analysis.

\section{Discussion}

We conducted this retrospective study to evaluate the role of CRS and NLR as independent biomarkers in advanced ovarian cancer patients treated with NACT and IDS. On 


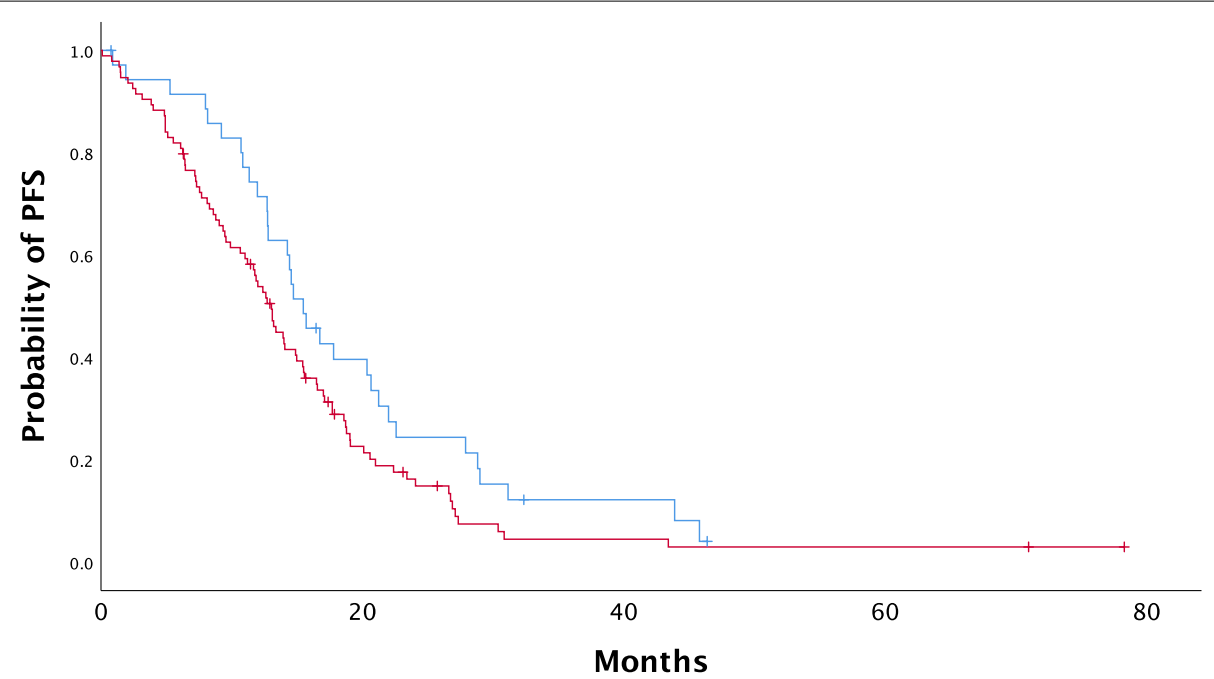

Fig. 1 Kaplan-Meier curves depicting progression-free survival (PFS) according to NLR status (blue line:NLR-low, red line: NLR-high)

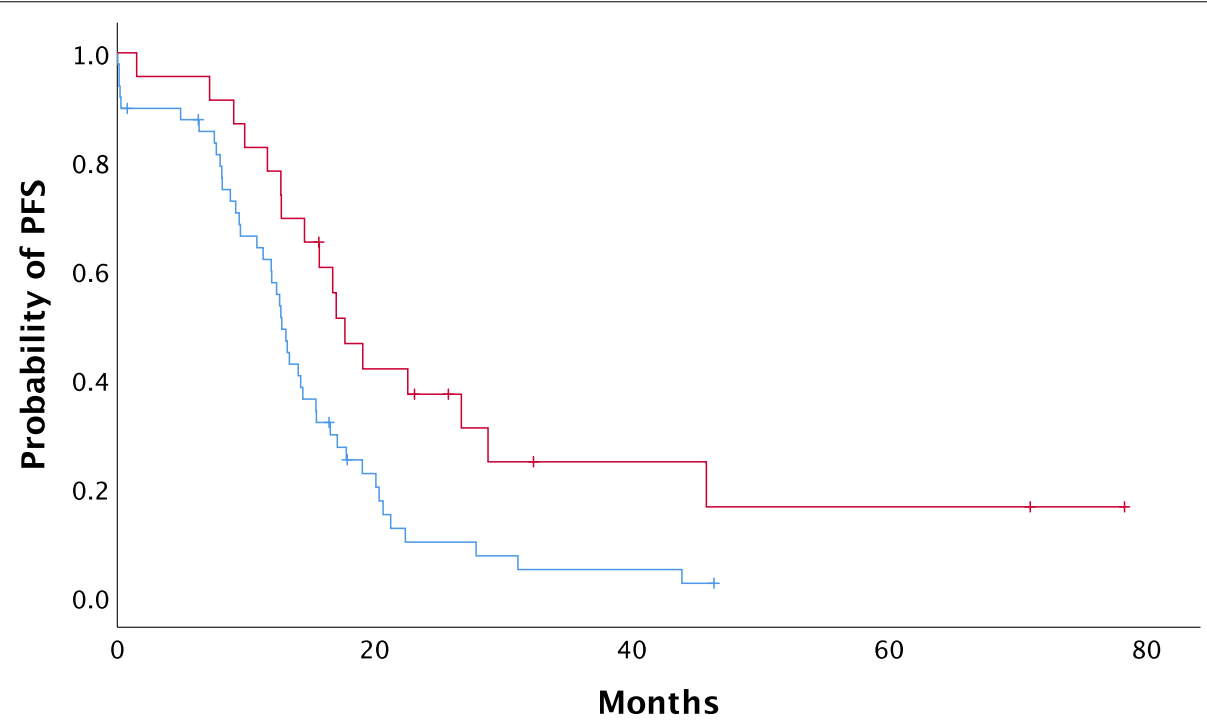

Fig. 2 Kaplan-Meier curves depicting progression-free survival (PFS) according to CRS (blue line: CRS1/2, red line: CRS3)

univariate analysis, NLR (high NLR $>3$ vs low NLR $\leq 3$ ) was significantly associated with OS $(P=0.012)$. In addition, NLR inversely correlated with mPFS, although the association was not statistically significant $(P=0.128)$. PFS was 14.56 months in patients with low NLR values prior to chemotherapy initiation and 12.39 months in those with high NLR values. Although CRS3 score was significantly associated with prolonged $\mathrm{mPFS}$ (CRS1/2: 12.79 months vs CRS3: 17.7 months; $P=0.008$ ), this correlation was not confirmed with $\mathrm{mOS}(P=0.876)$. Neither of these biomarkers was significantly associated with PFS or OS on multivariate analysis.

Systemic inflammation caused by malignancies is often expressed as neutrophilia, thrombocytosis and relative lymphocytopenia in the peripheral blood $[45,46]$. Neutrophils contribute to the development of an inflammatory microenvironment, promoting tumor growth, vascularization and metastasis. In addition, neutrophils 


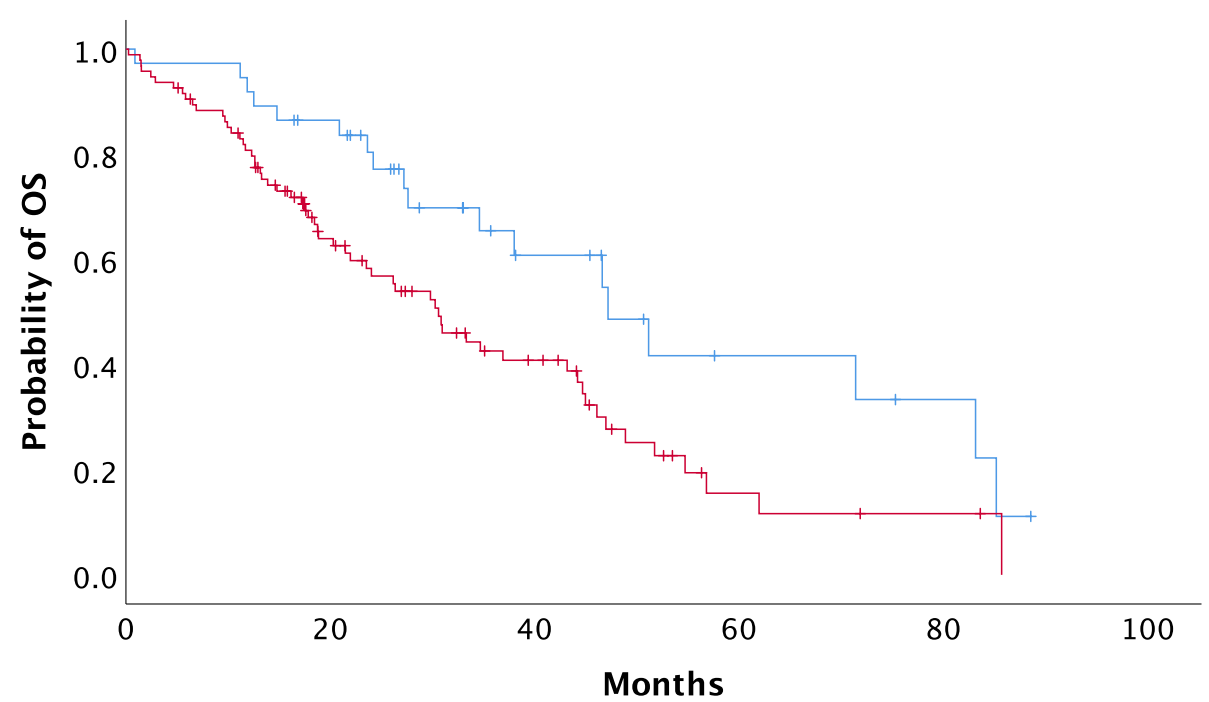

Fig. 3 Association between OS and NLR status. (blue line: NLR-low, red line: NLR-high)



Fig. 4 Association of chemotherapy response score (CRS) with overall survival. (blue line: CRS1/2, red line: CRS3)

attenuate the immune system by suppressing CD8+ T lymphocyte antitumor response through the release of nitric oxide synthase (iNOS) or arginase 1 (ARG1) [47]. Neutrophils facilitate tumor initiation and proliferation by producing MMP9 and transferring neutrophil elastase to cancer cells. In ovarian cancer patients, neutrophils possess intensified functions such as increased reactive oxygen species (ROS) production and increased adhesive ability [48] and may be implicated in disease progression by spreading to the omentum as early as before metastasis becomes detectable [49]. Neutrophils become abundant in the omentum and facilitate ovarian cancer cell implantation. Simultaneously, lower counts of lymphocytes may be related to the existence of a primary immunodeficiency. Lymphocytopenia may imply a poor lymphocyte-mediated immune response to malignancy and therefore an increased possibility of tumor recurrence. In this context, NLR was used as a representative index of inflammation and was evaluated in ovarian cancer. Interestingly, NLR has been proposed as useful marker for the preoperative discrimination of ovarian masses [50-52]. High NLR ratio could serve as an accurate discriminator of malignant ovarian masses in the preoperative setting. 
Table 2 Multivariate analysis for PFS

\begin{tabular}{llll}
\hline & $P$ Value & \multicolumn{2}{l}{$\mathbf{9 5 \% \mathrm { Cl }}$} \\
\cline { 3 - 4 } & & Lower & Upper \\
\hline $\begin{array}{l}\text { Bevacizumab adminis- } \\
\text { tration }\end{array}$ & .275 & .466 & 1.243 \\
ECOG score & .323 & .355 & 1.406 \\
Surgery & .001 & 1.704 & 6.973 \\
Age $>$ 65 & .413 & .769 & 1.893 \\
NLR & .903 & .618 & 1.724 \\
FIGO stage & .229 & .836 & 1.044 \\
\hline
\end{tabular}

Table 3 Multivariate analysis for OS

\begin{tabular}{llll}
\hline & $P$ Value & \multicolumn{2}{l}{$\mathbf{9 5 \% \mathrm { Cl }}$} \\
\cline { 3 - 4 } & & Lower & Upper \\
\hline $\begin{array}{llll}\text { Bevacizumab adminis- } \\
\text { tration }\end{array}$ & .134 & .309 & 1.169 \\
ECOG score & .271 & 719 & 3.231 \\
Surgery & .008 & 1.287 & 5.201 \\
Age $>$ 65 & .732 & .632 & 1.921 \\
NLR & .569 & .632 & 2.302 \\
FIGO stage & .784 & .859 & 1.122 \\
\hline
\end{tabular}

In ovarian cancer, the prognostic significance of NLR has been shown in several studies $[26,42,44,53]$ including metanalyses encompassing studies in either gynecological cancers or exclusively ovarian carcinomas [54]. In all the above referenced studies, increased NLR values were associated with worse prognosis and resistance to platinum-based therapy. It should be noted though that different cut-off limits were evaluated in these studies that enrolled mainly patients treated with primary debulking surgery.

Our study enrolled solely patients that underwent IDS after receiving three cycles of NACT. We report that NLR is associated with OS, but not PFS on univariate analysis. There is only a limited number of studies addressing this specific population. Kim et al. were the first to evaluate the predictive value of pretreatment NLR in ovarian cancer patients undergoing neoadjuvant chemotherapy in an Asian population [43]. In accordance with our findings, an elevated NLR defined as over 3.81 was independently associated with worse OS, but not PFS [43]. Of note, there was a significant association between PFS and the dynamic change in NLR during neoadjuvant chemotherapy (HR, 2.07; 95\% CI, 1.32-3.25). A similar study used a cut-off value of 6 and again increased NLR was significantly associated with worse OS in univariate analysis (HR 1.06, 95\% CI 1.01-1.13, $P=0.040$ ) but not in multivariate analysis (HR 1.05, 95\% CI 0.99-1.12, $P=0.080$ ) [26]. There was also no significant association between NLR and PFS in this population $(P=0.084)$. Finally, a sub analysis of MITO 24 study aimed to evaluate if an elevated NLR (defined as NLR $>3$ alike our study) could associate with clinical outcome [42]. The study included both patients treated with PDS and patients undergoing NACT and IDS approach. Elevated NLR was independently associated with PFS $(\mathrm{HR}=1.23$, 95\% CI 1.10-1.37), OS (HR $=1.41,95 \%$ CI $1.23-1.62)$ and PFI at 6 months $(\mathrm{OR}=2.52,95 \% \mathrm{CI} 1.30-4.87, p=0.006)$ and 12 months $(\mathrm{OR}=2.05,95 \% \mathrm{CI} 1.05-4.01, p=0.036)$ [42]. Interestingly, NLR lost its predictive value within the bevacizumab-treated population. In our study we have included both patients that received bevacizumab and patients that did not. Bevacizumab administration was significantly associated with PFS and OS, but this association lost its significance in the multivariate analysis indicating that bevacizumab administration is not an independent determinant of survival in these patients. In addition, the groups of patients that received or not bevacizumab in our study were heterogenous and further evaluation of NLR predictive value within each one lacks clinical significance. Overall, our results are in agreement with those observed in other studies addressing women treated with NACT and IDS.

Apart from NLR, other systemic inflammatory response markers have gained attention during the past years. Platelet-to-lymphocyte ratio (PLR) has been proposed as an indicator of prognosis in epithelial ovarian cancer in numerous studies $[55,56]$. Fibrinogen, as a key factor of coagulation cascade, also implicates solid tumors and cancer aggressiveness. Yang et al recently proposed the combination of preoperative fibrinogen and NLR (F-NLR) as a novel prognostic index in ovarian cancer although not in patients treated with IDS [41]. The F-NLR showed greater sensitivity and specificity than did fibrinogen or NLR alone. C-reactive protein (CRP) has been validated as a predictive factor in solid tumors [57]. Moreover, lymphocyte to monocyte ratio has been related with ovarian cancer aggressiveness and clinical outcome in a number of studies [58,59]. Collectively, NLR could be combined with other systemic markers to increase the accuracy achieved.

Apart though from its prognostic significance, NLR would be of value in ovarian cancer patients undergoing NACT, if it could predict response to chemotherapy as assessed currently by CRS. Bohm et al. initially demonstrated the prognostic significance of CRS score in terms of PFS and platinum sensitivity [11]. This initial observation was followed by others including our work validating the prognostic and/or predictive value of CRS in ovarian cancer patients undergoing NACT $[15,18,20,60,61]$. 
Recently, a metanalysis of published studies have shown that optimal response to NACT - designated as CRS3 - is associated with both FPS and OS benefit and this could be determined by the molecular biology of the disease as CRS3 was more frequently encountered among BRCA1/2 mutant patients [23]. However, this is not supported by other studies $[62,63]$ while other parameters as is the extent of the disease at initial diagnosis should be taken into consideration when evaluating response to chemotherapy $[22,64,65]$. In our study, no specific association between CRS and NLR at diagnosis was noted. Analogous was the result in the previous study conducted in the Asian population [43]. The number of patients could be a limiting factor for both studies. However, it should be taken into consideration whether this negative finding could really reflect the lack of a biological association. In our previous analysis, increased lymphocytic infiltration was correlated to CRS denoting that the inflammatory milieau of the tumor could determine sensitivity to chemotherapy [19]. This observation was in accordance with the prognostic role of tumor-infiltrating lymphocytes in ovarian cancer [66] corresponding probably to NLR-low tumors. Therefore, larger studies are warranted to fully evaluate this association.

Finally, it should be noted that there is a great diversity regarding the cut-off values of NLR among studies [40]. This raises the question of the biological significance between NLR-high and low populations. Median cutoff for high NLR was 2.95 among studies evaluating OS and 2.79 among studies evaluating PFS in correlation with NLR [40]. NLR threshold applied ranged between 0.89 to 5.03 across different studies. On that basis, we used 3 as the cut-off value and defined NLR $>3$ as high NLR value in our understudy population. However, it is evident that $72 \%$ of patients in our study were characterized as NLR-high by this binary classification. Obviously, these patients could not represent exclusively a population with adverse prognostic features. More likely, these patients designated as NLR-low in our study correspond to a population with minimal systemic inflammation and better prognostic features. This is most probably the reason that NLR lost its prognostic significance in the multivariate analysis.

Our study has some limitations. Firstly, the data was retrospectively collected and comprise a relatively small patient cohort from a single center. Moreover, there is a bias against ovarian cancer patients that could not eventually undergo IDS. Women with higher NLR values were more frequently unable to undergo debulking surgery than those with low NLR (21 vs 8 cases respectively). Although surgery was excluded from multivariate analysis, it may have still affected univariate analysis of NLR with PFS and OS. Finally, our study is based mainly on high grade ovarian cancer patients that could not be upfront debulked. Some studies support that the prognostic value of NLR may be greater in low or intermediate grade disease [40].

\section{Conclusions}

In conclusion, NLR could serve as a useful predictor of OS but not PFS in ovarian cancer patients receiving neoadjuvant treatment. High NLR defined as over 3 may indicate patients with worse prognosis. CRS score should be implemented in everyday clinical practice as a binary prognostication system to stratify ovarian patients at high risk for relapse. Both these biomarkers require further prospective validation, while their combination in normograms along with other common prognostic factors could improve stratification of ovarian cancer patients undergoing NACT. This could be a useful tool to guide future clinical trial design and treatment personalization.

\section{Acknowledgments \\ Not Applicable.}

\section{Authors' contributions}

Conceptualization: Michalis Liontos; Data curation: Michalis Liontos, Angeliki Andrikopoulou, Christos Markellos, Koutsoukos Konstantinos, Kaparelou Maria, Fiste Oraianthi, Skafida Efthymia, Haidopoulos Dimitris, Thomakos Nikolaos, Rodolakis Alexandros, Formal analysis: Angeliki Andrikopoulou, Christos Markellos, Kaparelou Maria, Fiste Oraianthi, Methodology: Michalis Liontos, Christos Markellos, Koutsoukos Konstantinos, Kaparelou Maria, Fiste Oraianthi, Skafida Efthymia, Project administration; Michalis Liontos, Haidopoulos

Dimitris, Thomakos Nikolaos, Rodolakis Alexandros, Zagouri Flora, Dimopoulos Meletios-Athanasios, Supervision: Liontos Michalis, Zagouri Flora, Dimopoulos Meletios-Athanasios, Writing - original draft: Liontos Michalis, Angeliki Andrikopoulou, Writing - review \& editing: Liontos Michalis, Angeliki Andrikopoulou. The author(s) read and approved the final manuscript.

\section{Funding}

This research received no external funding.

\section{Availability of data and materials}

Data presented in our study can be found in the patients' archives that are safely stored in our Institution. The datasets generated during the current study are available from the corresponding author upon request.

\section{Declarations}

Ethics approval and consent to participate

Informed consent was obtained from all individual participants included in the study. The study has been performed in accordance with the 1964 Helsinki Declaration and has been approved by the Institutional Review Board of Alexandra University Hospital.

Consent for publication

Written informed consent has been obtained from all the patients enrolled to publish this paper.

\section{Competing interests}

Dr. Liontos has received honoraria by Roche, Astra Zeneca, Astellas, MSD, Janssen, BMS and IPSEN, Dr. Koutsoukos has received honoraria by Roche, BMS, MSD and IPSEN, Dr. Zagouri receiving honoraria by Novartis, Eli-Lilly, Merck, Pfizer, and Roche Dr. Dimopoulos has received honoraria by Janssen, Celgene, Takeda, Amgen, Genesis Pharma and BMS.Dr. Andrikopoulou, Dr. Kaparelou, 
Dr. Markellos, Dr. Fiste, Dr. Skafida, Dr. Thomakos, Dr. Haidopoulos and Dr. Rodolakis declare no conflict of interest.

\section{Author details}

${ }^{1}$ Department of Clinical Therapeutics, Alexandra General Hospital, School of Medicine, National and Kapodistrian University of Athens, Athens, Greece. ${ }^{2}$ Department of Obstetrics and Gynecology, Alexandra General Hospital, School of Medicine, National and Kapodistrian University of Athens, Athens, Greece.

Received: 30 June 2021 Accepted: 13 October 2021

Published online: 01 November 2021

\section{References}

1. Bray F, Ferlay J, Soerjomataram I, Siegel RL, Torre LA, Jemal A. Global cancer statistics 2018: GLOBOCAN estimates of incidence and mortality worldwide for 36 cancers in 185 countries. CA Cancer J Clin. 2018:68:394-424

2. Cancer Tomorrow. https://gco.iarc.fr/tomorrow/graphic-isotype?type= 1\&type_sex $=0 \&$ mode $=$ population $\&$ sex $=2 \&$ populations $=900 \&$ cance $r s=25 \& a g e \_g r o u p=v a l u e \& a p c \_m a l e=0 \& a p c \_f e m a l e=0 \&$ single_unit $=$ 50000\&print $=0$. Accessed 4 May 2020.

3. Prat J. New insights into ovarian cancer pathology. Ann Oncol. 2012. https://doi.org/10.1093/annonc/mds300.

4. Matulonis UA, Sood AK, Fallowfield L, Howitt BE, Sehouli J, Karlan BY. Ovarian cancer. Nat Rev Dis Prim. 2016;2:1-22.

5. Vergote I, Tropé CG, Amant F, et al. Neoadjuvant chemotherapy or primary surgery in stage IIIC or IV ovarian cancer. N Engl J Med. 2010;363:943-53.

6. Onda T, Satoh T, Saito T, et al. Comparison of treatment invasiveness between upfront debulking surgery versus interval debulking surgery following neoadjuvant chemotherapy for stage III/IV ovarian, tubal, and peritoneal cancers in a phase III randomised trial: Japan Clinical Oncology Group Study JCOG0602. Eur J Cancer. 2016;64:22-31.

7. Liu EL, Mi RR, Wang DH, Wang LQ, Zhang YM, Chen W. Application of combined intraperitoneal and intravenous neoadjuvant chemotherapy in senile patients with advanced ovarian cancer and massive ascites. Eur J Gynaecol Oncol. 2017;38:209-13.

8. Pölcher M, Mahner S, Ortmann O, et al. Neoadjuvant chemotherapy with carboplatin and docetaxel in advanced ovarian cancer - a prospective multicenter phase II trial (PRIMOVAR). Oncol Rep. 2009;22:605-13.

9. van der Burg MEL, van Lent M, Buyse M, Kobierska A, Colombo N, Favalli $G$, et al. The effect of debulking surgery after induction chemotherapy on the prognosis in advanced epithelial ovarian cancer. N Engl J Med. 1995:332:629-34.

10. Cooke SL, Brenton JD. Evolution of platinum resistance in high-grade serous ovarian cancer. Lancet Oncol. 2011;12:1169-74.

11. Böhm S, Faruqi A, Said I, et al. Chemotherapy response score: development and validation of a system to quantify histopathologic response to neoadjuvant chemotherapy in tubo-ovarian high-grade serous carcinoma. J Clin Oncol. 2015;33:2457-63.

12. Böhm S, Le N, Lockley M, Brockbank E, Faruqi A, Said I, et al. Histopathologic response to neoadjuvant chemotherapy as a prognostic biomarker in tubo-ovarian high-grade serous carcinoma: updated chemotherapy response score (CRS) results. Int J Gynecol Cancer. 2019;29:353-6.

13. Glenn Mccluggage W, Judge MJ, Clarke BA, et al. Data set for reporting of ovary, fallopian tube and primary peritoneal carcinoma: recommendations from the International Collaboration on Cancer Reporting (ICCR). Mod Pathol. 2015;28:1101-22.

14. Said I, Böhm S, Beasley J, et al. The chemotherapy response score (CRS): Interobserver reproducibility in a simple and prognostically relevant system for reporting the histologic response to neoadjuvant chemotherapy in Tuboovarian high-grade serous carcinoma. Int J Gynecol Pathol. 2017;36:172-9.

15. Ditzel HM, Strickland KC, Meserve EE, et al. Assessment of a chemotherapy response score (CRS) system for tubo-ovarian high-grade serous carcinoma (HGSC). Int J Gynecol Pathol. 2019;38:230-40.

16. Santoro A, Angelico G, Piermattei A, et al. Pathological chemotherapy response score in patients affected by high grade serous ovarian carcinoma: the prognostic role of omental and ovarian residual disease. Front Oncol. 2019. https://doi.org/10.3389/fonc.2019.00778.

17. Williams AT, Ganesan R. Role of the pathologist in assessing response to treatment of ovarian and endometrial cancers. Histopathology. 2020;76:93-101.

18. Coghlan E, Meniawy TM, Munro A, et al. Prognostic role of histological tumor regression in patients receiving neoadjuvant chemotherapy for high-grade serous tubo-ovarian carcinoma. Int J Gynecol Cancer. 2017;27:708-13.

19. Liontos M, Sotiropoulou M, Kaparelou M, et al. Lymphocytic infiltration and chemotherapy response score as prognostic markers in ovarian cancer patients treated with neoadjuvant chemotherapy. Gynecol Oncol. 2020;157:599-605.

20. Lee JY, Chung YS, Na K, Kim HM, Park CK, Nam EJ, et al. External validation of chemotherapy response score system for histopathological assessment of tumor regression after neoadjuvant chemotherapy in tuboovarian high-grade serous carcinoma. J Gynecol Oncol. 2017. https://doi. org/10.3802/jgo.2017.28.e73.

21. Rajkumar S, Polson A, Nath R, Lane G, Sayasneh A, Jakes A, et al. Prognostic implications of histological tumor regression (Böhm's score) in patients receiving neoadjuvant chemotherapy for high grade serous tubal \& ovarian carcinoma. Gynecol Oncol. 2018;151:264-8.

22. Petrillo M, Zannoni GF, Tortorella L, Pedone Anchora L, Salutari V, Ercoli A, et al. Prognostic role and predictors of complete pathologic response to neoadjuvant chemotherapy in primary unresectable ovarian cancer. Am J Obstet Gynecol. 2014;211:632.e1-8.

23. Cohen PA, Powell A, Böhm S, et al. Pathological chemotherapy response score is prognostic in tubo-ovarian high-grade serous carcinoma: a systematic review and meta-analysis of individual patient data. Gynecol Oncol. 2019;154:441-8.

24. Templeton AJ, McNamara MG, Šeruga B, et al. Prognostic role of neutrophil-to-lymphocyte ratio in solid tumors: a systematic review and meta-analysis. J Natl Cancer Inst. 2014. https://doi.org/10.1093/jnci/ dju124.

25. Chen G, Zhu L, Yang Y, Long Y, Li X, Wang Y. Prognostic role of neutrophil to lymphocyte ratio in ovarian cancer: a meta-analysis. Technol Cancer Res Treat. 2018. https://doi.org/10.1177/1533033818791500.

26. Salman L, Sabah G, Jakobson-Setton A, Raban O, Yeoshoua E, Eitan R. Neutrophil-to-lymphocyte ratio as a prognostic factor in advanced stage ovarian carcinoma treated with neoadjuvant chemotherapy. Int J Gynecol Obstet. 2020;148:102-6.

27. Jeerakornpassawat D, Suprasert P. Potential predictors for chemotherapeutic response and prognosis in epithelial ovarian, fallopian tube and primary peritoneal cancer patients treated with platinum-based chemotherapy. Obstet Gynecol Sci. 2020;63:55-63.

28. Baert T, Van Camp J, Vanbrabant L, Busschaert P, Laenen A, Han S, et al. Influence of CA125, platelet count and neutrophil to lymphocyte ratio on the immune system of ovarian cancer patients. Gynecol Oncol. 2018;150:31-7.

29. Badora-Rybicka A, Nowara E, Starzyczny-Słota D. Neutrophil-to-lymphocyte ratio and platelet-to-lymphocyte ratio before chemotherapy as potential prognostic factors in patients with newly diagnosed epithelial ovarian cancer. ESMO Open. 2016. https://doi.org/10.1136/esmoopen-2016-000039.

30. YildizY, Kucukzeybek Y, Alacacioglu A, et al. Prognostic value of preoperative neutrophil-to-lymphocyte ratio and platelet-to-lymphocyte ratio in patients with epithelial ovarian cancer. Eur J Gynaecol Oncol. 2017;38:444-8.

31. Komura N, Mabuchi S, Yokoi E, Kozasa K, Kuroda H, Sasano T, et al. Comparison of clinical utility between neutrophil count and neutrophil-lymphocyte ratio in patients with ovarian cancer: a single institutional experience and a literature review. Int J Clin Oncol. 2018;23:104-13.

32. Zhou M, Li L, Wang X, Wang C, Wang D. Neutrophil-to-lymphocyte ratio and platelet count predict long-term outcome of stage IIIC epithelial ovarian cancer. Cell Physiol Biochem. 2018;46:178-86.

33. Wu YY, Qin YY, Qin JQ, Zhang X, Lin FQ. Diagnostic value of derived neutrophil-to-lymphocyte ratio in patients with ovarian cancer. J Clin Lab Anal. 2019. https://doi.org/10.1002/jcla.22833.

34. Chen L, Wang X, Shu J, Xu S, Wu Q, Yu Y. Diagnostic value of serum D-dimer, CA125, and neutrophil-to-lymphocyte ratio in differentiating ovarian cancer and endometriosis. Int J Gynecol Obstet. 2019;147:212-8.

35. Yildirim M, Cendek BD, Avsar AF. Differentiation between benign and malignant ovarian masses in the preoperative period using neutrophil-to-lymphocyte and platelet-to-lymphocyte ratios. Mol Clin Oncol. 2015;3:317-21. 
36. Aotsuka A, Matsumoto Y, Arimoto T, et al. Interleukin-17 is associated with expression of programmed cell death 1 ligand 1 in ovarian carcinoma. Cancer Sci. 2019;110:3068-78.

37. Sanguinete MMM, De Oliveira PH, Martins-Filho A, Micheli DC, TavaresMurta BM, Murta EFC, et al. Serum IL-6 and IL-8 correlate with prognostic factors in ovarian cancer. Immunol Investig. 2017;46:677-88.

38. Santos ID, Jammal MR, Silveira TP, Murta EFC, Nomelini RS. Stromal IL2 is related to the neutrophil/lymphocyte ratio in epithelial ovarian cancer. Pathologica. 2019;111:62-6.

39. Marchetti C, Romito A, Musella A, Santo G, Palaia I, Perniola G, et al. Combined plasma fibrinogen and neutrophil lymphocyte ratio in ovarian cancer prognosis may play a role? Int J Gynecol Cancer. 2018;28:939-44.

40. Ethier JL, Desautels DN, Templeton AJ, Oza A, Amir E, Lheureux S. Is the neutrophil-to-lymphocyte ratio prognostic of survival outcomes in gynecologic cancers? A systematic review and meta-analysis. Gynecol Oncol. 2017;145:584-94

41. Yang J, Ma J, Cheng S, Wang Y. The combination of plasma fibrinogen concentration and neutrophil lymphocyte ratio (F-NLR) as a prognostic factor of epithelial ovarian cancer. Onco Targets Ther. 2020;13:7283-93.

42. Farolfi A, Scarpi E, Greco F, et al. Inflammatory indexes as predictive factors for platinum sensitivity and as prognostic factors in recurrent epithelial ovarian cancer patients: a MITO24 retrospective study. Sci Rep. 2020;10:1-8.

43. Kim YJ, Lee I, Chung YS, Nam EJ, Kim S, Kim SW, et al. Pretreatment neutrophil-to-lymphocyte ratio and its dynamic change during neoadjuvant chemotherapy as poor prognostic factors in advanced ovarian cancer. Obstet Gynecol Sci. 2018;61:227-34.

44. Williams KA, Labidi-Galy SI, Terry KL, Vitonis AF, Welch WR, Goodman A, et al. Prognostic significance and predictors of the neutrophil-to-lymphocyte ratio in ovarian cancer. Gynecol Oncol. 2014;132:542-50.

45. Grivennikov SI, Greten FR, Karin M. Immunity, inflammation, and cancer. Cell. 2010;140:883-99.

46. Bamias A, Gavalas NG, Karadimou A, Dimopoulos MA. Immune response in ovarian cancer: how is the immune system involved in prognosis and therapy: potential for treatment utilization. Clin Dev Immunol. 2010. https:// doi.org/10.1155/2010/791603.

47. Ocana A, Nieto-Jiménez C, Pandiella A, Templeton AJ. Neutrophils in cancer: prognostic role and therapeutic strategies. Mol Cancer. 2017. https://doi. org/10.1186/s12943-017-0707-7.

48. Klink M, Jastrzembska K, Nowak M, Bednarska K, Szpakowski M, Szyllo K, et al. Ovarian cancer cells modulate human blood neutrophils response to activation in vitro. Scand J Immunol. 2008;68:328-36.

49. Lee WJ, Ko SY, Mohamed MS, Kenny HA, Lengyel E, Naora H. Neutrophils facilitate ovarian cancer premetastatic niche formation in the omentum. J Exp Med. 2019;216:176-94.

50. Eo WK, Kim KH, Park EJ, Kim HY, Kim HB, Koh SB, et al. Diagnostic accuracy of inflammatory markers for distinguishing malignant and benign ovarian masses. J Cancer. 2018:9:1165-72.

51. Yildirim MA, Seckin KD, Togrul C, Baser E, Karsli MF, Gungor T, et al. Roles of neutrophil/lymphocyte and platelet/lymphocyte ratios in the early diagnosis of malignant ovarian masses. Asian Pacific J Cancer Prev. 2014;15:6881-5.

52. Bakacak M, Serin S, Ercan Ö, Köstü B, Bostancı MS, Bakacak Z, et al. Utility of preoperative neutrophil-to-lymphocyte and platelet-to-lymphocyte ratios to distinguish malignant from benign ovarian masses. J Turkish Ger Gynecol Assoc. 2016;17:21-5.

53. Cho H, Hur HW, Kim SW, Kim SH, Kim JH, Kim YT, et al. Pre-treatment neutrophil to lymphocyte ratio is elevated in epithelial ovarian cancer and predicts survival after treatment. Cancer Immunol Immunother. 2009:58:15-23.

54. Yang Z, Gu JH, Guo CS, Li XH, Yang WC. Preoperative neutrophil-tolymphocyte ratio is a predictor of survival of epithelial ovarian cancer: a systematic review and meta-analysis of observational studies. Oncotarget. 2017:8:46414-24.

55. Asher V, Lee J, Innamaa A, Bali A. Preoperative platelet lymphocyte ratio as an independent prognostic marker in ovarian cancer. Clin Transl Oncol. 2011;13:499-503.

56. Raungkaewmanee S, Tangjitgamol S, Manusirivithaya S, Srijaipracharoen S, Thavaramara T. Platelet to lymphocyte ratio as a prognostic factor for epithelial ovarian cancer. J Gynecol Oncol. 2012;23:265-73.

57. Shrotriya S, Walsh D, Nowacki AS, Lorton C, Aktas A, Hullihen B, et al. Serum c-reactive protein is an important and powerful prognostic biomarker in most adult solid tumors. PLoS One. 2018. https://doi.org/10.1371/journal. pone.0202555.

58. Eo WK, Chang HJ, Kwon SH, et al. The lymphocyte-monocyte ratio predicts patient survival and aggressiveness of ovarian cancer. J Cancer. 2016;7:289-96

59. Cai L, Song Y, Zhao X. Prognostic significance of lymphocyte monocyte ratio in patients with ovarian cancer. Med (United States). 2020. https://doi.org/ 10.1097/MD.0000000000019638.

60. Michaan N, Chong WY, Han NY, Lim MC, Park SY. Prognostic value of pathologic chemotherapy response score in patients with ovarian cancer after neoadjuvant chemotherapy. Int J Gynecol Cancer. 2018;28:1676-82.

61. Lawson BC, Euscher ED, Bassett RL, Liu J, Ramalingam P, Zhong Y, et al. A 3-tier chemotherapy response score for ovarian/fallopian tube/peritoneal high-grade serous carcinoma: is it clinically relevant? Am J Surg Pathol. 2020:44:206-13.

62. Lee YJ, Kim HS, Rim JH, Lee JY, Nam EJ, Kim SW, et al. Germline BRCA, chemotherapy response scores, and survival in the neoadjuvant treatment of ovarian cancer. BMC Cancer. 2020. https://doi.org/10.1186/s12885-020-6688-8.

63. Barrington DA, Felix AS, Owda R, et al. Pathologic chemotherapy response score in epithelial ovarian cancer: surgical, genetic, and survival considerations. Surg Oncol. 2020;34:40-5.

64. Sassen S, Schmalfeldt B, Avril N, Kuhn W, Busch R, Höfler H, et al. Histopathologic assessment of tumor regression after neoadjuvant chemotherapy in advanced-stage ovarian cancer. Hum Pathol. 2007;38:926-34

65. Liang M, Prendergast E, Staples J, Holschneider C, Cass I. Complete pathologic response at interval debulking surgery following neoadjuvant chemotherapy predicts improved survival in women with advanced epithelial ovarian cancer in a multi-institutional cohort. Gynecol Oncol. 2016;143:197.

66. Zhang L, Conejo-Garcia JR, Katsaros D, et al. Intratumoral T cells, recurrence, and survival in epithelial ovarian cancer. N Engl J Med. 2003;348:203-13.

\section{Publisher's Note}

Springer Nature remains neutral with regard to jurisdictional claims in published maps and institutional affiliations.
Ready to submit your research? Choose BMC and benefit from:

- fast, convenient online submission

- thorough peer review by experienced researchers in your field

- rapid publication on acceptance

- support for research data, including large and complex data types

- gold Open Access which fosters wider collaboration and increased citations

- maximum visibility for your research: over 100M website views per year

At BMC, research is always in progress.

Learn more biomedcentral.com/submissions 